13

\title{
Структурные свойства углеродных пленок, полученных методом ионно-стимулированного импульсно-плазменного осаждения в атмосфере азота
}

\author{
(С) И.А. Завидовский, О.А. Стрелецкий, О.Ю. Нищак, Н.Ф. Савченко, С.В. Дворяк, А.В. Павликов
}

Московский государственный университет им. М.В. Ломоносова,

119991 Москва, Россия

e-mail: ia.zavidovskii@physics.msu.ru

Поступило в Редакцию 5 июня 2019 г.

В окончательной редакции 21 сентября 2019 г.

Принято к публикации 25 сентября 2019 г.

\begin{abstract}
Углеродные пленки, полученные методом импульсно-плазменного ионно-стимулированного распыления графита в атмосфере смеси аргона и азота, были исследованы при помощи КР-спектроскопии, электронной дифракции и РФЭС. Согласно полученным данным, азот эффективно встраивается в структуру материала, что приводит к формированию ориентированных графитовых нанокластеров, доля которых растет с увеличением энергии стимуляции.
\end{abstract}

Ключевые слова: аморфный углерод, тонкие пленки, ионная стимуляция, КР-спектроскопия, рентгеновская фотоэлектронная спектроскопия.

DOI: $10.21883 / J T F .2020 .03 .48937 .232-19$

\section{Введение}

Свойства углеродных материалов в значительной мере зависят от содержания атомов углерода, имеющих различную гибридизацию. В аморфных углеродных пленках могут присутствовать все типы гибридизаций, свойственные углероду [1].

Модификация пленок при помощи внедрения в их структуру донорных или акцепторных атомов используется для создания покрытий, сочетающих высокие прочностные свойства и хорошую проводимость. Благодаря этим качествам азотосодержащие углеродные пленки могут использоваться в качестве покрытия жестких дисков [2], эмиссионных электродов [3], АСМ-кантилеверов [4]. Также подобные пленки могут наноситься на полимеры, служащие для упаковки, в таком случае они могут выступать в качестве газового барьера [5].

Тем не менее анализ соединений, которые образует азот, встраиваясь в углеродную матрицу, показывает, что его влияние на структуру не сводится только к ее „легированию“" [6].

Роль азота в формировании структуры аморфных углеродных пленок была подробно исследована для покрытий, получаемых в процессе химического осаждения из газовой фазы (CVD). Данный процесс может осуществляться в различных режимах, например, при высокочастотном плазменном разложении смеси $\mathrm{C}_{6} \mathrm{H}_{6} / \mathrm{N}_{2}$ [7], осаждении смеси $\mathrm{Ar} / \mathrm{CH}_{4} / \mathrm{N}_{2}$ [8], разложении смеси $\mathrm{CH}_{4} / \mathrm{N}_{2}$, усиленном плазмой электрон-циклотронного резонанса [9]. В работах по CVD-синтезу показано, что в зависимости от условий осаждения встраивание азота может приводить к формированию различных химических связей, и, соответственно, к изменениям электрофизических и механических свойств получаемых пленок.

Значительно менее изученным методом синтеза углеродных покрытий, который широко исследуется в настоящее время, является импульсно-плазменное распыление графита [10-15]. В число его преимуществ входит возможность осуществлять послойное осаждение наноструктур и синтез метастабильных фаз углерода, а также отсутствие необходимости охлаждения образцов, позволяющее осуществлять напыление на широкий спектр подложек. Особый интерес представляют азотированные пленки, полученные при помощи импульсноплазменного осаждения, которые обладают хорошими механическими и трибологическими свойствами [11]. Разнообразие применений азотированных углеродных пленок и различные эффекты, обусловленные внедрением азота, влекут за собой необходимость исследования их структуры и роли азота в ее формировании.

В настоящей работе пленки, синтезированные методом импульсно-плазменного ионно-стимулированного напыления, были изготовлены при различных энергиях стимуляции ионным пучком и исследованы методами спектроскопии комбинационного рассеяния (КР-спектроскопии), просвечивающей электронной микроскопии (ПЭМ), электронной дифракции, рентгеновской фотоэлектронной спектроскопии (РФЭС).

\section{1. Получение образцов и методы их исследования}

Исследуемые образцы изготавливались методом импульсно-плазменного ионно-стимулированного распыле- 
ния графита. Напыление осуществлялось в атмосфере смеси аргона и азота при давлении $10^{-1} \mathrm{~Pa}$. Относительная концентрация азота в рабочей атмосфере не изменялась и составляла $25 \%$. Время импульса разряда составляло порядка $1 \mathrm{~ms}$, частота следования импульсов $1 \mathrm{~Hz}$, мощность разряда $1 \mathrm{~kW}$. В качестве подложек использовались фольга Ті ВТ1-0 $(98.28 \%$ Ti) и скол монокристалла $\mathrm{NaCl}$. Перед напылением было осуществлено травление подложек пучком ионов $\mathrm{Ar}^{+}$энергией $1000 \mathrm{eV}$. Температура подложек во время процесса осаждения не превышала $50^{\circ} \mathrm{C}$. В ходе процесса напыления при помощи холловского ионного источника осуществлялась стимуляция роста пленок ионами различных энергий (от 200 до $700 \mathrm{eV}$ ) и фиксированном токе. Толщина образцов, измеренная при помощи ПЭМ, уменьшалась от 72 до $26 \mathrm{~nm}$ с ростом энергии стимуляции.

Образцы были исследованы методами спектроскопии комбинационного рассеяния света (КР-спектроскопии), просвечивающей электронной микроскопии (ПЭМ), рентгеновской фотоэлектронной спектроскопии (РФЭС).

КР-спектры были измерены на спектрометре Horiba HR 800 с длиной волны гелий-неонового лазера $632.8 \mathrm{~nm}$, мощностью $1 \mathrm{MW}$ и разрешающей способностью $1 \mathrm{~cm}^{-1}$.

Исследования с помощью просвечивающей электронной микроскопии осуществлялись на базе просвечивающего электронного микроскопа LEO 912 ab с ускоряющим напряжением $120 \mathrm{keV}$.

РФЭС-спектры были получены с помощью электронного спектрометра Kratos AXIS Ultra DLD.

Рентгеновское излучение в спектрометре обеспечивалось источником с магниевым анодом с энергией, соответствующей дублету $\mathrm{Al} K_{\alpha}=1486.6 \mathrm{eV}$.

\section{2. Экспериментальные результаты}

\section{1. КР-спектроскопия}

На рис. 1 представлены характерные КР-спектры исследованных образцов. Согласно [16], подобные спектры характерны для аморфных углеродных структур с преимущественным содержанием атомов с $s p^{3}$-гибридизацией.

Пики, максимумы которых расположены на частотах $1370 \mathrm{~cm}^{-1}$ (D-линия) и $1540 \mathrm{~cm}^{-1}$ (G-линия), характерны для большинства углеродных структур. $D$-линия свидетельствует о наличии дефектов или оборванных связей в исследуемой структуре, а $G$-линия является признаком $s p^{2}$-гибридизованных атомов [16]. В случае алмазоподобных пленок рост $D$-линии означает возрастание доли разупорядоченной графитовой компоненты. Такой эффект, сопровождающийся изменением спектров, может проявляться при различной модификации образцов, например, при их отжиге $[17,18]$.

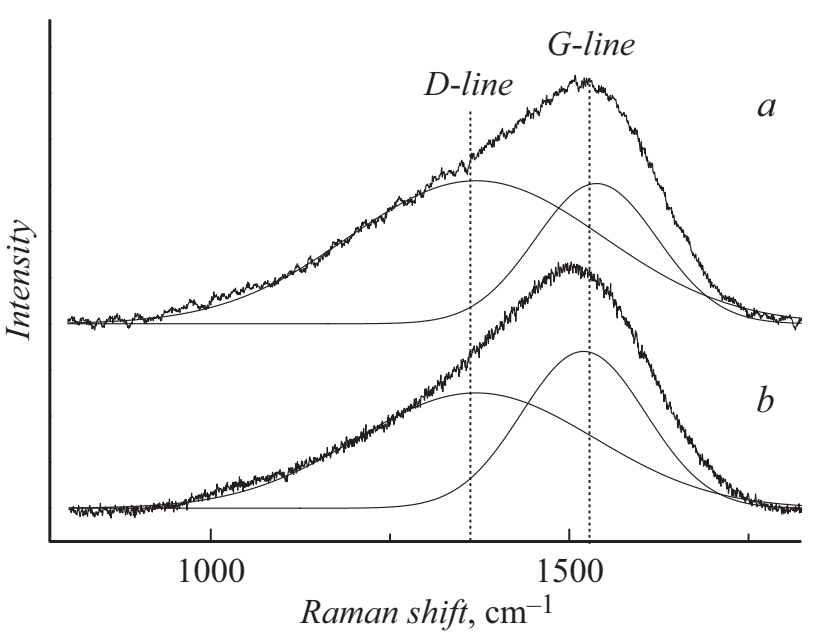

Рис. 1. КР-спектры образцов: $a-$ энергия стимуляции $400 \mathrm{eV}$, $b-$ без ионной стимуляции.

Анализ спектров исследуемых образцов показывает, что происходит незначительное увеличение интенсивности и уширение $D$-линии. Это косвенно свидетельствует о том, что при ионной стимуляции наблюдается образование графитовых нанокластеров, имеющих сильную дисперсию по размерам.

\section{2. Электронная дифракция}

Разупорядоченные алмазоподобные структуры, как правило, имеют в своей дифракционной картине два широких гало, отвечающих межплоскостным расстояниям 0.206 и $0.115 \mathrm{~nm}$ [19-21]. Данные линии наблюдались в дифракции образца, полученного без ионной стимуляции (рис. $2, a$ ). На рис. $2, b$ изображена дифракционная картина образца, полученного в условиях стимуляции ионами энергии $400 \mathrm{eV}$. Помимо линий 0.206 и $0.115 \mathrm{~nm}$ он характеризуется наличием линии $0.335 \mathrm{~nm}$, свидетельствующей о наличии в образце графитовой фазы [19].

Дифракция образца, полученного при энергии ассистирования $400 \mathrm{eV}$, является характерной для всех образцов, полученных в условиях ионной стимуляции. Анализ дифракционных картин показал, что относительная интенсивность линии, соответствующей межплоскостному расстоянию графита, увеличивалась с ростом энергии стимуляции. Это может являться свидетельством увеличения доли графитовых кластеров в структуре пленки.

Особенностью дифракционных картин пленок, изготовленных в условиях ионной стимуляции, является наличие дуг линии $0.335 \mathrm{~nm}$. На рис. 3 приведена зависимость интенсивности данной линии графита от азимутального угла. Изменение интенсивности данной линии составляет до $30 \%$ от максимума. Согласно литературным данным [22,23], данная особенность свидетельствует о наличии преимуществен- 

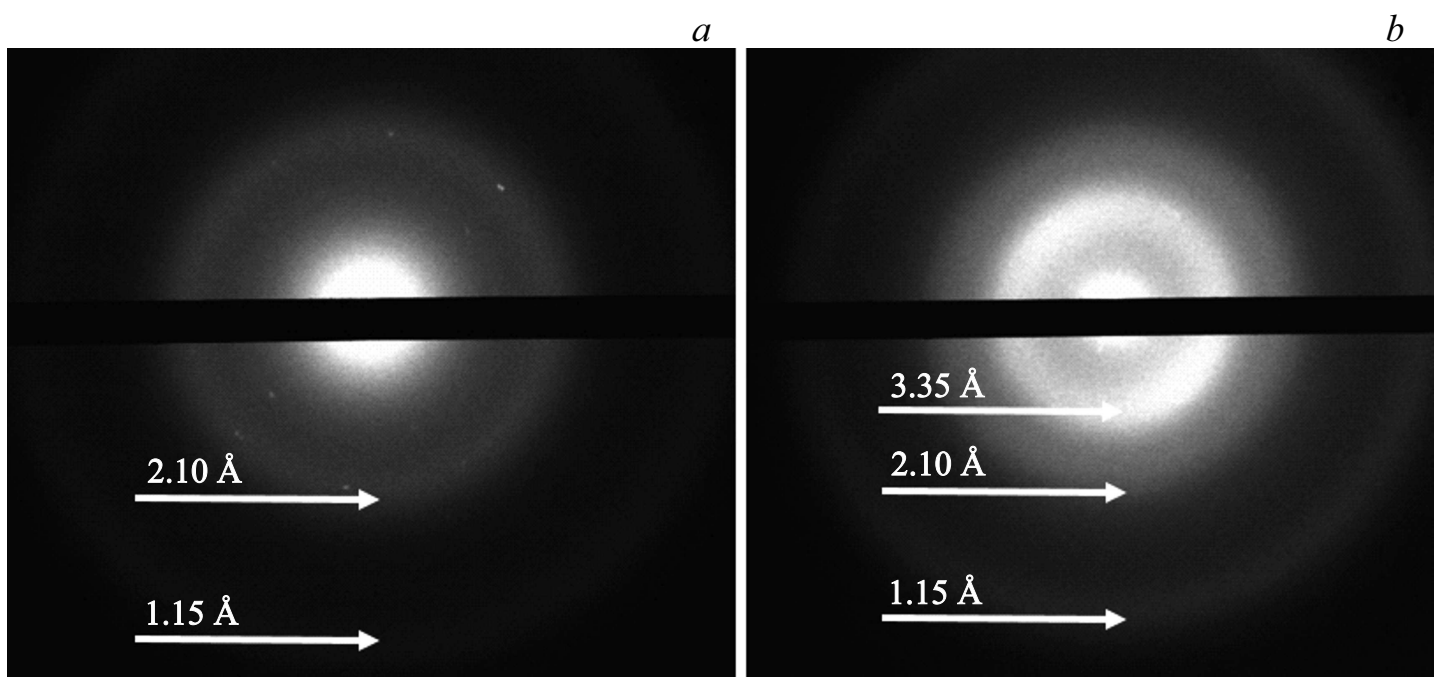

Рис. 2. Дифракционные картины образцов, нанесенных без ионной стимуляции $(a)$ и при энергии ионной стимуляции $400 \mathrm{eV}(b)$.
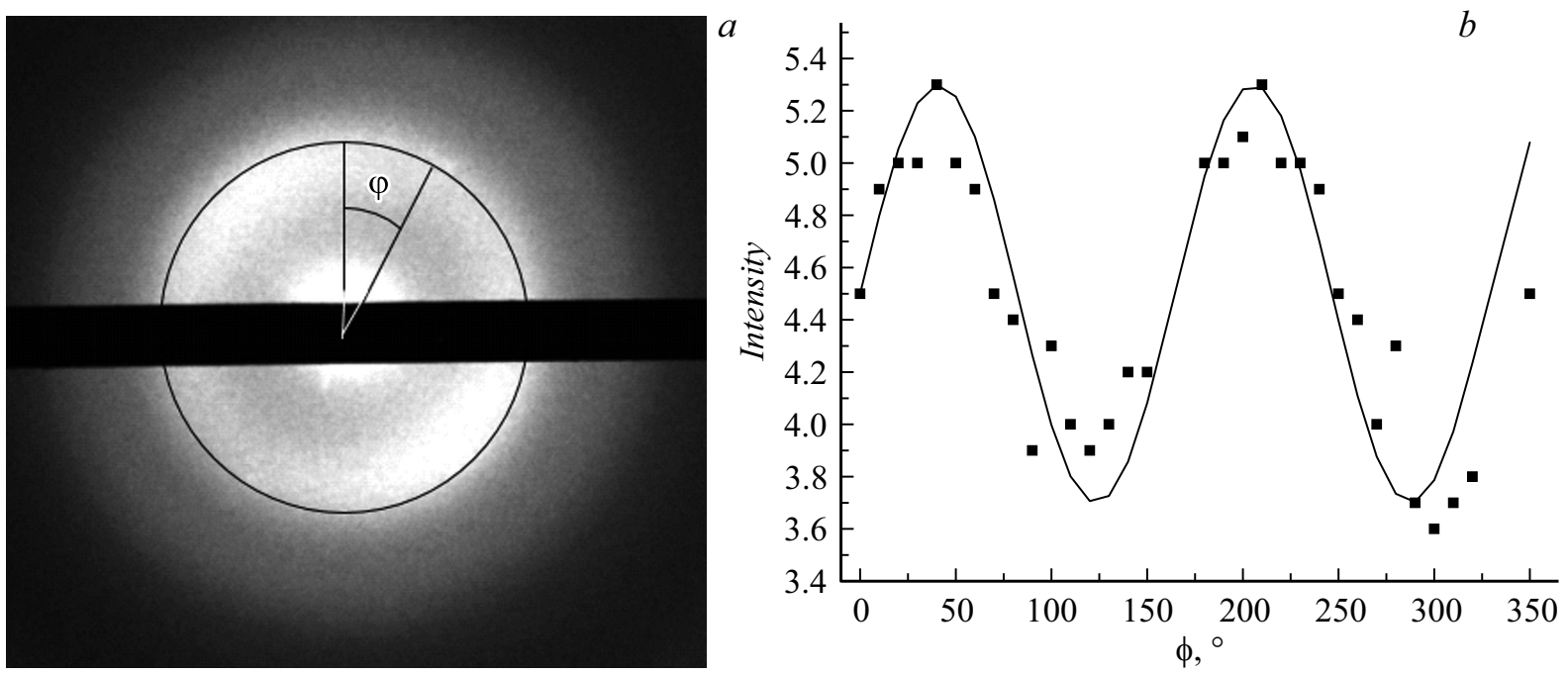

Рис. 3. Дифракционная линия $3.35 \AA$ (a) и зависимость ее интенсивности от азимутального угла $(b)$.

ной ориентации графитовых кристаллитов вдоль направления ионного облучения. Подобный эффект наблюдается при ионной стимуляции поликристаллических пленок [24-26]. В ходе формирования структуры происходит более эффективная перестройка тех кристаллитов, направление плотной упаковки которых не совпадает с углом падения пучка, тогда как для кристаллитов, ориентированных в направлении пучка, проявляется эффект каналирования. Вследствие этого осаждаемая структура приобретает преимущественную ориентацию.

Таким образом, согласно данным электронной дифракции, введение стимуляции в процесс нанесения аморфных пленок привело к появлению ориентированных графитовых кристаллов в их структуре. Увеличение линии $0.335 \mathrm{~nm}$ коррелирует с увеличением энергии ассистирующих ионов.

\section{3. РФЭС}

В таблице приведены данные по элементному составу исследуемых структур, полученные из их обзорных РФЭС-спектров. При увеличении энергии стимуляции в элементном составе углеродных пленок возрастает доля азота.

На рис. 4 приведен спектр C1s и N1s линий образца, изготовленного при энергии стимуляции $400 \mathrm{eV}$. Для других образцов, полученных в условиях ионной стимуляции, относительные интенсивности линий данного спектра отличаются незначительно.

Наибольшей интенсивностью в рассматриваемом спектре обладает линия $\mathrm{C}\left(s p^{3}\right)-\mathrm{C}$ [27], что говорит о высокой доле алмазных связей в аморфной фазе образца. Гораздо меньшей интенсивностью обладает линия $\mathrm{C}=\mathrm{O}$. Ее наличие обусловлено окислением поверхностного 
Элементный состав пленок, нанесенных при различной энергии ионной стимуляции

\begin{tabular}{c|c|c|c}
\hline \multicolumn{4}{c}{ Элементный состав пленок } \\
\hline $\begin{array}{c}\text { Энергия } \\
\text { стимуляции }\end{array}$ & $200 \mathrm{~V}$ & $400 \mathrm{~V}$ & $600 \mathrm{~V}$ \\
\hline $\mathrm{C}$ & $79.3 \%$ & $82.2 \%$ & $65 \%$ \\
\hline $\mathrm{N}$ & $5 \%$ & $5.9 \%$ & $10.2 \%$ \\
\hline $\mathrm{O}$ & $11.4 \%$ & $9 \%$ & $23.9 \%$ \\
\hline Другое & $4.3 \%$ & $2.9 \%$ & $0.9 \%$
\end{tabular}

слоя образца, поскольку глубина анализа метода РФЭС составляет $0.5-3 \mathrm{~nm}[28]$.

Положение линии $\mathrm{C}-\mathrm{N}-286.6 \mathrm{eV}$ в соответствии с литературными данными [29] свидетельствует о том, что азот связан с $s p^{2}$-гибридизованным углеродом (связи $\mathrm{C}\left(s p^{3}\right)-\mathrm{N}$ соответствует большая энергия $287.2-287.5 \mathrm{eV}$ [27]). Также на это указывает положение линии N1s - $399.8 \mathrm{eV}$, соответствующее связи $\mathrm{N}-\mathrm{C}\left(s p^{2}\right)$ [29] или $\mathrm{N}=\mathrm{C}\left(s p^{2}\right)$ [30]. Наличие линии $\mathrm{C}\left(s p^{2}\right)-\mathrm{C}$ на $284.9 \mathrm{eV}$ подтверждает данные электронной дифракции и КР-спектроскопии, свидетельствующие об образовании графитовой фазы в структуре пленки.

Полученные данные показывают, что увеличение числа графитовых кластеров, проявившееся в виде увеличения интенсивности $D$-линии спектра комбинационного рассеяния и дифракционной линии $0.335 \mathrm{~nm}$, вызвано эффективной перестройкой структуры под действием ионной стимуляции. На рис. 4 показана предполагаемая схема включения атомов азота в структуру, приводящая к увеличению доли $s p^{2}$-фазы.

Особенностью спектров также является наличие сателлитов линии $\mathrm{C} 1 \mathrm{~s}$, соответствующих потерям энергии

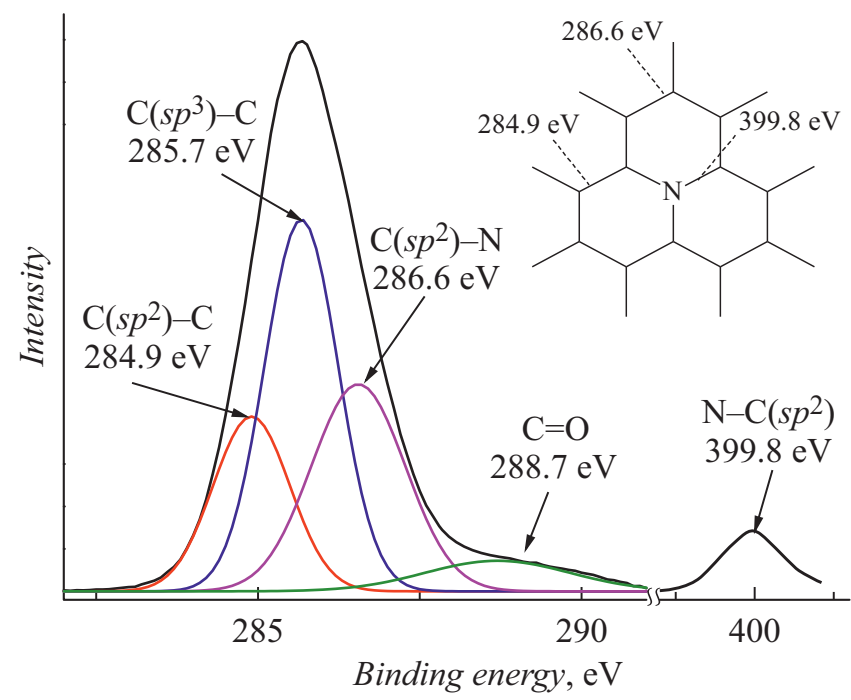

Рис. 4. РФЭС-спектр C1s и N1s линии образца, полученного при энергии стимуляции $400 \mathrm{eV}$.

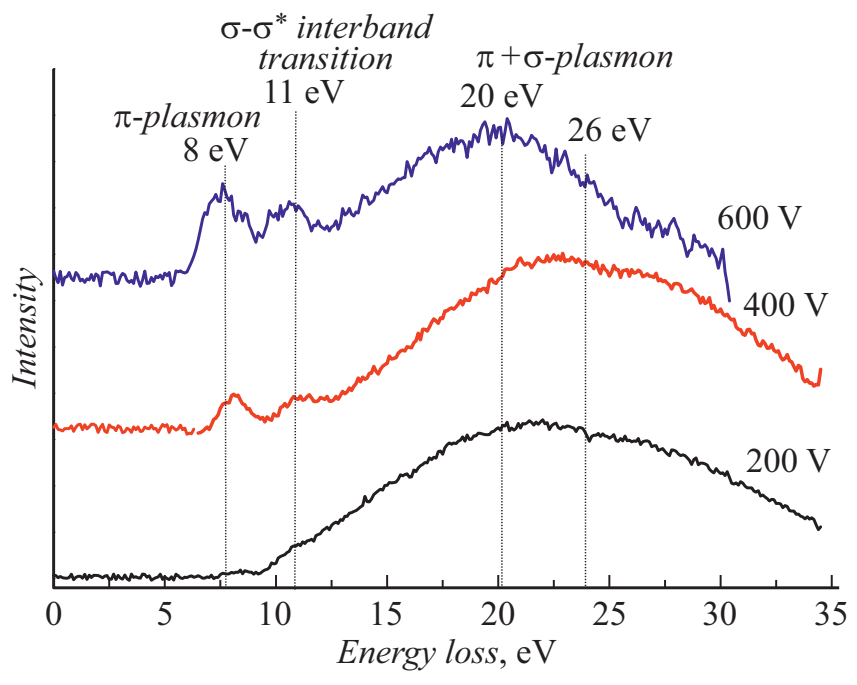

Рис. 5. Спектр потерь энергии электронов для образцов, нанесенных при различных энергиях стимуляции.

фотоэлектронов. Спектры потерь энергии фотоэлектронов представлены на рис. 5. При этом нуль энергии отсчитывается от положения максимума C1s-линии.

Согласно [31], в углеродных структурах наблюдается пик с энергией 5-10 eV, характеризующий потери на возбуждение $\pi$-плазмона (коллективного возбуждения $\pi$-электронов структуры), и пик, энергия которого составляет $20 \mathrm{eV}$ и более, отвечающий колебаниям всей электронной подсистемы $(\pi+\sigma$-плазмон $)$.

Пик, отвечающий энергии $10-11 \mathrm{eV}$, согласно [32,33], соответствует межзонному переходу типа $\sigma-\sigma^{*}$, который характерен как для алмазоподобных, так и для графитоподобных структур. Также данный пик можно интерпретировать как $\pi$-подобный плазмон, однако, согласно [34,35], он характерен для фуллереновых структур. Таким образом, для исследуемых образцов данный пик, скорее всего, обусловлен наличием межзонного перехода.

На спектрах образцов можно отметить сложную форму пика $\pi+\sigma$-плазмона, состоящего из двух линий, центры которых имеют положение примерно $20 \mathrm{eV}$ и 26-27 eV. Линия 26-27 eV [36] может быть интерпретирована как возбуждение объемного $\pi+\sigma$-плазмона графита. В свою очередь, $20 \mathrm{eV}$ - энергия поверхностного плазмона, которая меньше энергии объемного в $\sqrt{ } 2$ раз. С увеличением энергии стимуляции интенсивность линии поверхностного плазмона возрастает, что может свидетельствовать о росте соотношения поверхности графитовых кластеров к их объему, т.е. об уменьшении размеров графитовых кристаллитов.

\section{Заключение}

В работе исследованы пленки, изготовленные методом импульсно-плазменного ионно-стимулированного 
распыления графита в атмосфере смеси аргона и азота. Согласно данным электронной дифракции, введение ионной стимуляции в процесс напыления привело к появлению графитовой фазы в структуре пленок, причем было также установлено, что ее доля возрастает с увеличением энергии стимуляции. Данные РФЭС позволяют утверждать, что увеличение энергии ассистирующих ионов также приводит к их более эффективному встраиванию в структуру и возрастанию доли азота в составе пленки до 10\%. При этом анализ C1s линии показывает, что атомы азота преимущественно связаны с $s p^{2}$-гибридизованными атомами в структуре пленки. Таким образом, внедрение ионов азота приводит к эффективной перестройке структуры и появлению ориентированных графитовых нанокластеров в составе пленки.

\section{Конфликт интересов}

Авторы заявляют, что у них нет конфликта интересов.

\section{Список литературы}

[1] Chu P.K., Li L. // Mater. Chem. Phys. 2006. Vol. 96. N 2-3. P. 253.

[2] Casiraghi C., Robertson J., Ferrari A.C. // Mater. Today. 2007. Vol. 10. N 1-2. P. 44.

[3] Amaratunga G.A.J., Silva S.R.P. // Appl. Phys. Lett. 1996. Vol. 68. N 18. P. 2529

[4] Poplavsky A.I., Kolpakov A.Y., Kudriavtsev Y., Asomoza R., Goncharov I.Yu., Galkina M.E., Manokhin S.S., Kharchenko V.A. // Vacuum. 2018. Vol. 152. P. 193.

[5] Ray S.C., Mukherjee D., Sarma S., Bhattacharya G., Mathur A., Roy S.S., Mc Laughlin J.A. // Diamond Relat. Mater. 2017. Vol. 80. P. 59.

[6] Silva S.R.P., Robertson J., Amaratunga G.A.J., Rafferty B., Brown L.M. // J. Appl. Phys. 1997. Vol. 81. N 6. P. 2626.

[7] Yamamoto S., Kawana A., Ichimura H., Masuda C. // Surf. Coat. Technol. 2012. Vol. 210. P. 1.

[8] Tsuchiya M., Murakami K., Magara K., Nakamura K., Ohashi H., Tokuda K., Takami T., Ogasawara H., Enta Y., Suzuki Y., Ando S., Nakazawa H. // Jpn. J. Appl. Phys. 2016. Vol. 55. N 6. P. 065502.

[9] Seker Z., Ozdamar H., Esen M., Esen R., Kavak H. // Appl. Surf. Sci. 2014. Vol. 314. P. 46.

[10] Hu J., Tian Q., Wan P., Chen B., Tian X., Gong C., Golosov D.A. // Diamond and Related Mater. 2019. Vol. 98. P. 107479.

[11] Streletskiy O.A., Zavidovskiy I.A., Nischak O.Y., Pavlikov A.V. // Thin Solid Films. 2018. Vol. 671. P. 31.

[12] Poplavsky A.I., Kolpakov A.Y., Kudriavtsev Y. et al.// Vacuum. 2018. Vol. 152. P. 193.

[13] Volosova M. // Key Engineer. Mater. 2016. Vol. 723. P. 262.

[14] Corbella C., Portal S., Zolotukhin D.B., Martinez L.G., Lin L., Kundrapu M., Keidar M. // Plasma Sources Sci. Technol. 2019. Vol. 28. P. 4.

[15] Kolpakov A.Y., Poplavsky A.I., Galkina M.E., Sudzhanskaya I.V., Goncharov I.Yu., Druchinina O.A., Strigunov N.V., Kharchenko V.A., Merchansky O.Yu. // Nanotechnol. Russia. 2010. Vol. 5. N 3-4. P. 160-164.
[16] Ferrari A., Robertson J. // Phil. Trans. R. Soc. Lond. 2004. A. Vol. 362. P. 2477.

[17] Mominuzzaman S.M., Krishna K.M., Soga T., Jimbo T., Umeno M. // Carbon. 2000. Vol. 38. N 1. P. 127.

[18] Ogwu A., Lamberton R., Morley S., Maguire P., Mc Laughlin J. // Physica B: Condensed Matter. 1999. Vol. 269. N 3-4. P. 335.

[19] Wang Q., Wang C., Wang Z., Zhang J., He D. // Appl. Phys. Lett. 2007. Vol. 91. N 14. P. 141902.

[20] Voevodin A.A., Jones J.G., Zabinski J.S., Czigány Z., Hultman L. // J. Appl. Phys. 2002. Vol. 92. N 9. P. 4980.

[21] Neidhardt J., Hultman L., Czigány Z. // Carbon. 2004. Vol. 42. P. 2729.

[22] Moafi A., Partridge J.G., Sadek A.Z., Mc Culloch D.G. // J. Mater. Chem. A. 2012. Vol. 1. N 2. P. 402. DOI: $10.1039 / \mathrm{c} 2 \mathrm{ta00198 \textrm {e }}$

[23] Shiell T.B., McCulloch D.G., Bradby J.E., Haberl B., Boehler R., Mc Kenzie D. R. // Scientific Reports. 2016. Vol. 6. P. 37232.

[24] Jiang X., Zhang W.J., Klages C.-P. // Phys. Rev. B. 1998. Vol. 58. N 11. P. 7064.

[25] Zhang L., Tong S., Zhang L., Tong S., Liu H., Li Y., Wang Z. // Mater. Lett. 2016. Vol. 171. P. 304. // Mater. Lett. 2016. Vol. 171. P. 304.

[26] Cuomo J.J., Rossnagel S.M., Kaufman H.R. Handbook of Ion Beam Processing Technology: Principles, Deposition, Film Modification and Synthesis, Noyes Publications, Westwood, New Jersey, USA. 1989. P. 175-176.

[27] Chattopadhyay S., Chen L.C., Wu C.T., Chen K.H., Wu J.S., Chen Y.F., Lehmann J., Hess. P. // Appl. Phys. Lett. 2001. Vol. 79. N 3. P. 332. DOI:10.1063/1.1386619

[28] Seah M.P. // Vacuum. 1984. Vol. 34. N 3-4. P. 463.

[29] Li X., Yang S., Wu X. // J. Univer. Sci. Technol. Beijing, Mineral, Metallurgy, Mater. 2006. Vol. 13. N 3. P. 272.

[30] Sivkov A., Pak A., Shanenkov I., Kolganova J., Shatrova K. // IOP Conference Series: Materials Science and Engineering. 2014. Vol. 66. P. 012001.

[31] Бржсезинская М.М., Байтингер Е.М., Смирнов А.Б. // ФTT. 2006. Vol. 48. P. 5.

[32] Logothetidis S. // Diamond Related Mater. 2003. Vol. 12. N 2. P. 141.

[33] Calliari L., Fanchenko S., Filippi M. // Carbon. 2007. Vol. 45. N 7. P. 1410.

[34] Leiro J.A., Heinonen M.H., Laiho T., Batirev I.G. // J. Electron. Spectr. Related Phenomena. 2003. Vol. 128. P. 205.

[35] Östling D., Apell P., Rosén A. // EPL (Europhys. Lett.) 1992. Vol. 21. P. 5.

[36] Filippi M., Calliari L. // Surf. Interf. Anal. 2006. Vol. 38. N 4. P. 595. 